\section{Fast pulsars with disks}

\section{F. C. Michel}

Space Physics and Astronomy Department, Rice University, Houston, Texas 77251, USA

\section{A. J. Dessler}

Space Science Laboratory, NASA Marshall Space Flight Center, Huntsville, Alabama 35812, USA

It is quite likely that fast pulsars are not rare; there are several point-like radio sources with pulsar-like radio spectra that might prove to pulse in the milisecond range (J. H. Taylor, personal communication). The possibility that the recently discovered pulsar ${ }^{1}$ PSR1937 +214 is a new class of object has been considered by adherents of the vacuum model ${ }^{2,3}$. We point out here that the measured parameters of PSR1937 + 214 are consistent with the proposed disk model of radio pulsars ${ }^{4,5}$. Using the measured values of $\boldsymbol{P}$ and $\dot{P}$ and the scalings provided in this theory ${ }^{5}$, we expect a surface magnetic field strength of $\sim 10^{8} \mathrm{G}$, a radio-emission output of $10^{31} \mathrm{erg} \mathrm{s}^{-1}$, and a weak (below detection with present instruments) output of energy in the $X$-ray and $\gamma$-ray ranges. The surface magnetic field cannot be checked, but the observed radio luminosity ${ }^{1}$ is $3 \times 10^{30} \mathrm{erg}$ $\mathrm{s}^{-1}$, in gratifying agreement with the theoretical expectation of the disk model.

In our model ${ }^{4,5}$, an isolated magnetized rotating neutron star is encircled by a massive $\left(\sim 10^{-5} \boldsymbol{M}_{\odot}\right)$ fluid disk. Relative rotation between the disk and neutron star induces potential differences across the disk, and between the disk and neutron star as in a Faraday disk dynamo, permitting a return of the polar cap current from the disk to the star by auroral arcing. Pulsed emission arises from the return current flow to preferential regions of the star controlled by its surface magnetic field structure ${ }^{4}$.

In the disk model, the predicted average radio luminosity ${ }^{5}$ is related to $P$ and $\dot{P}$ by

$$
L_{\mathrm{rad}}=10^{30} \mathrm{erg} \mathrm{s}^{-1}\left(\dot{P} / 10^{-18}\right)(10 / P)^{8 / 3}
$$

where $P$ is in milliseconds. The expected luminosity is $10^{31} \mathrm{erg} \mathrm{s}^{-1}$ for $1937+214$, compared with the observed value $\sim 3 \times 10^{30} \mathrm{erg} \mathrm{s}^{-1}$. The position of $1937+214$ relative to other pulsars is shown in Fig. 1, along with lines of constant radio luminosity from equation (1).

On average, pulsars have the luminosity indicated by equation (1), although there is considerable scatter (a factor of $\sim 30$ about the mean) as can be seen in Fig. 5 of ref. 5 . Consequently, the close agreement in this case is probably fortuitous. It is difficult to compare this prediction directly against the vacuum rotator models owing to recent changes forced by the failure of the aligned rotator $\operatorname{model}^{6}$, and also the tendency to concentrate on explaining only the Crab pulsar ${ }^{7}$ rather than pulsars as a class. In any event, PSR1937+214 would be considered anomalously dim in the vacuum model, because only about $10^{-6}$ of the energy output appears as radioemission, in contrast with the ratio of energy output to input of $10^{-3}$ typical of other pulsars ${ }^{8}$. As can be seen from Fig. 1, however, this pulsar has the luminosity expected by the disk model and is not anomalously dim.

According to the disk model, PSR1937 +214 should not be a strong emitter of $\mathrm{X}$ rays and $\gamma$ rays. The auroral zone potential drop, which gives the maximum potential available for accelerating particles, is $10^{10} \mathrm{~V}$ (see Fig. 4 of ref. 5). This is about average for pulsars in contrast to $10^{11}-10^{12} \mathrm{~V}$ across the auroral zone for the Crab and Vela pulsars ${ }^{5}$. The energetic photon flux emitted by curvature radiation scales as particle

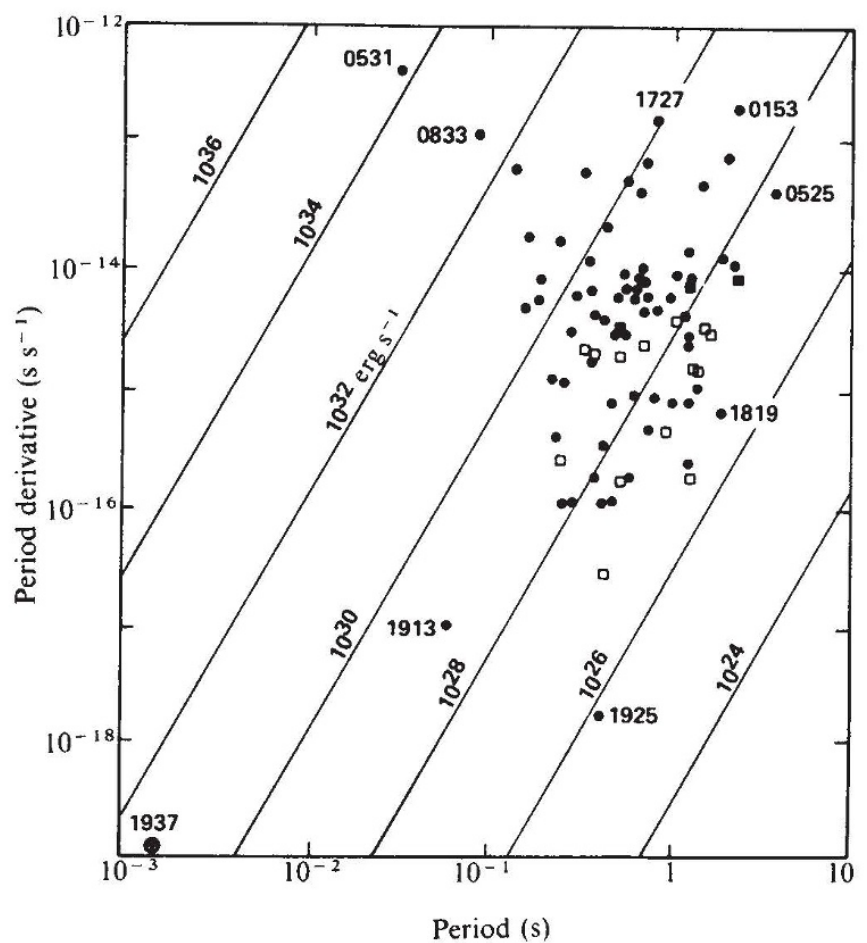

Fig. 1 Predicted radio luminosity of pulsars in the disk model (adapted from Fig. 3 of ref. 5).

energy to the fourth power, which may explain why only the Crab and Vela pulsars are bright sources of energetic photons.

In the disk model ${ }^{5}$, the surface polar magnetic field strength is given by

$$
B_{\text {surf }}=10^{10} \mathrm{G}(\dot{P} / 10)^{1 / 2}(P / 10)^{1 / 6}
$$

For $1937+214$, equation (2) indicates a surface field of $\sim 10^{8}$ $\mathrm{G}$. We should emphasize that this is the value of $B$ required to give the correct total power output from the neutron star, whereas equation (1) corresponds only to the radio power emitted.

Our magnetic field value of $10^{8} \mathrm{G}$ is slightly less than the $3 \times 10^{8} \mathrm{G}$ inferred by Alpar et $a l^{2}$ and Arons ${ }^{3}$. In the disk model, the disk forces the field lines to open and form a stellar wind at the co-rotation distance (roughly 3 neutron-star radii) rather than at the light-cylinder distance ( 8 radii). Therefore, a given torque is produced in the disk model with a weaker surface field than in the vacuum models; the typical pulsar magnetic field is only $10^{10} \mathrm{G}$ in the disk model compared with $10^{12} \mathrm{G}$ in the vacuum models ${ }^{9}$. Consequently, a field of the order of $10^{8} \mathrm{G}$ is less surprising on the basis of the disk model, although it is an unusually weakly magnetized object. (We do not discuss here the issue of neutron star magnetization.)

We conclude that PSR $1937+214$ is not necessarily a new class of pulsar but that its properties are consistent with the disk model and do not require special explanation, although it may have had a peculiar evolutionary history ${ }^{1-3}$.

We thank R. Elsner, M. Weisskopf, J. Fishman and R. Wilson for helpful criticism. F.C.M. acknowledges support for this research from NASA under grant NAGW 379.

Received 26 January; accepted 8 March 1983.

1. Backer, D. C., Kulkarni, S. R., Heiles, C., Davis, M. M. \& Goss, W. M. Nature 300, 615-618 (1982).

2. Alpar, M. A., Cheng, A. F., Ruderman, M. A. \& Shaham, J. Nature 300, 728-730 (1982).

3. Arons, J. Nature 302, 301-305 (1983).

4. Michel, F. C. \& Dessler, A. J. Astrophys. J. 251, 654-664 (1981).

5. Michel, F. C. Astrophys. J. 266, 188-200 (1983).

6. Fawley, W. M., Arons, J. \& Scharlemann, E. T. Astrophys. J. 217, 227-243 (1977)

7. Cheng, A. F. \& Ruderman, M. A. A strophys. J. 229, 348-360 (1979).

8. Manchester, R. N. \& Taylor, J. H. Pulsars, 112 (Freeman, San Francisco, 1977).

8. Manchester, R. N. \& Taylor, J. H. Pulsars, 1
9. Michel, F. C. Rev. Mod. Phys. 54, 1 (1982), 